\title{
Emergency department charges may be associated with mortality in patients with severe sepsis and septic shock: a cohort study
}

Nicholas M. Mohr ${ }^{1,2}$, Ryan Dick-Perez ${ }^{1,2^{*}}$ (D), Azeemuddin Ahmed ${ }^{1}$, Karisa K. Harland', Dan Shane ${ }^{3}$, Daniel Miller ${ }^{1}$, Christine Miyake ${ }^{4}$, Levi Kannedy ${ }^{1}$, Brian M. Fuller ${ }^{5}$ and James C. Torner ${ }^{6}$

\begin{abstract}
Background: Sepsis severity of illness is challenging to measure using claims, which makes sepsis difficult to study using administrative data. We hypothesized that emergency department (ED) charges may be associated with hospital mortality, and could be a surrogate marker of severity of illness for research purposes. The objective of this study was to measure concordance between ED charges and mortality in admitted patients with severe sepsis or septic shock.

Methods: Cohort study of all adult patients presenting to a 60,000-visit Midwestern academic ED with severe sepsis or septic shock (by ICD-9 codes) between July 1, 2008 and June 30, 2010. Data on demographics, admission APACHE-II score, and disposition was extracted from the medical record, and comorbidities were identified from diagnosis codes using the Elixhauser methodology. Summary statistics were reported and bivariate concordance was tested using Pearson correlation. Logistic regression models for 28-day mortality were developed to measure the independent association with mortality.

Results: We included a total of 294 patients in the analysis. We found that ED charges were inversely related to mortality (adjusted OR 0.829 per $\$ 1000$ increase in total ED charges, 95\%Cl 0.702-0.980). ED charges were also independently associated with 28-day hospital-free and ICU-free days (0.74 days increase per $\$ 1000$ additional ED charges, 95\%Cl 0.06-1.41 and 0.81 days increase per $\$ 1000$ additional ED charges, 95\%Cl 0.05-1.56, respectively). ED charges were also associated with APACHE-II score (\$34 total ED charges per point increase in APACHE-II score, 95\%Cl \$6-62).

Conclusions: ED charges in administrative data sets are associated with in-hospital mortality and health care utilization, likely related to both illness severity and intensity of early sepsis resuscitation. ED charges may have a role in risk adjustment models using administrative data for acute care research.
\end{abstract}

Keywords: Sepsis, Health services research, Costs and cost analysis, Risk adjustment, Emergency service, hospital, Critical illness

\footnotetext{
* Correspondence: Ryan.dickperez@gmail.com; Ryan-dick-perez@uiowa.edu

${ }^{1}$ Department of Emergency Medicine, University of lowa College of

Medicine, 200 Hawkins Drive, 1008 RCP, lowa City, IA 52246, USA

${ }^{2}$ Division of Critical Care, Department of Anesthesia, University of lowa Carver

College of Medicine, 200 Hawkins Dr. 6546 JCP, lowa City, IA 52246, USA

Full list of author information is available at the end of the article
}

(c) The Author(s). 2018 Open Access This article is distributed under the terms of the Creative Commons Attribution 4.0 International License (http://creativecommons.org/licenses/by/4.0/), which permits unrestricted use, distribution, and reproduction in any medium, provided you give appropriate credit to the original author(s) and the source, provide a link to the Creative Commons license, and indicate if changes were made. The Creative Commons Public Domain Dedication waiver (http://creativecommons.org/publicdomain/zero/1.0/) applies to the data made available in this article, unless otherwise stated. 


\section{Background}

Administrative data sets are an increasingly important tool for conducting population-based and health services research as they contain detailed information about medical care and outcomes [1]. Administrative data sets allow for rapid identification of a large cohort of patients, and they facilitate study across medical centers and health systems [2]. While health services studies using administrative data in sepsis and critical care exist [3, 4], it has been challenging to perform rigorous analyses because no robust physiologic measure of illness severity is captured in hospital claims [5]. Therefore there remains a need for a validated method to adjust for severity of illness based on administrative data.

Sepsis is responsible for $17 \%$ of US in-hospital deaths, and nearly one-half of sepsis admissions are treated in the emergency department (ED) [6, 7]. Age, medical comorbidities, admission diagnosis, and surgical status impact morbidity and mortality significantly, so risk adjustment models are critical to compare outcomes across clinical sepsis studies [8]. Illness severity scores, such as the Acute Physiology and Chronic Health Evaluation, 2nd edition (APACHE-II) score [9], the Simplified Acute Physiology Score (SAPS), and the Sequential Organ Failure Assessment (SOFA) score are valid scoring systems for critically ill patients [10-12], but the parameters used to calculate these scores are not captured in claims.

The objective of this study is to measure the concordance between ED charges and mortality in patients admitted with severe sepsis or septic shock, with a secondary objective of measuring the association between ED charges and APACHE-II score. We hypothesize that intensity of care is closely related to severity of illness, and that level of intensity could be approximated by ED charges. If validated, ED charges could be a useful covariate for claimsbased health services analyses of acute care conditions like sepsis.

\section{Methods}

\section{Study design}

This study was a cohort study of all adult (age $\geq 18$ years) patients presenting to a Midwestern academic 60,000-visit ED with severe sepsis or septic shock between July 1, 2008 and June 30, 2010. Severe sepsis and septic shock were defined by International Classification of Diseases, 9th Edition (ICD-9) hospital discharge diagnosis codes (995.92 or 785.52), evidence of infection during the index ED visit, and admission to the intensive care unit (ICU) during the hospital stay. Patients who had surgery performed were excluded from the data set. The study was approved by the local institutional review board (University of Iowa IRB-001, reference number 201211702) under waiver of informed consent.

\section{Study protocol}

After charts were identified by ICD-9 criteria, a study investigator $(\mathrm{CM})$ manually reviewed each medical record to confirm that the study subjects had infection in the ED. Then a research assistant, blinded to the study hypothesis, reviewed each medical record to abstract data using a standardized case report form. We collected data on demographics, source of infection, APACHE-II score at hospital admission, and final disposition. Two data sets were used in the study, clinical data to calculate the APACHE-II scores and administrative data set to extract ED charges and hospital discharge diagnosis codes.

Professional fees and hospital facility charges were maintained as separate variables. Comorbidities were defined from billing discharge diagnosis codes using the Elixhauser methodology, which defines a set of 30 conditions associated with clinical outcomes and health care utilization [13]. For calculation of APACHE-II scores, we used laboratory and vital sign values from the medical record, and we assumed laboratory values that were not measured were normal. We recorded hospital mortality and 28-day hospital-free and ICU-free days, which we defined as the number of days in the first 28 days after admission that a patient spent alive outside the hospital and the ICU, respectively. Using this measurement for length-of-stay is frequently done in studies of the critically ill to accurately account for censored data among non-survivors.

\section{Outcomes}

The primary outcome of the study was concordance between ED charges and mortality. Secondary outcomes included the association between ED charges, APACHE-II score, and Elixhauser comorbidities with hospital survival and hospital length-of-stay (measured as 28-day hospitalfree and ICU-free days).

\section{Analysis}

We performed summary statistics using the Student's t-test, the Wilcoxon rank-sum test, and the chi-squared test, as appropriate. We used Pearson correlation coefficient to describe bivariate concordance between ED charges and APACHE-II score. Each analysis was conducted with total ED charges, ED professional fees, and ED facility charges separately, and the charge with the strongest association with APACHE-II score was used in subsequent charge-based analyses.

Next, a logistic regression model was developed using the primary outcome of hospital mortality. Three separate models were constructed and compared using the Bayesian Information Criterion (BIC): one had APACHE-II score as a single predictor, the second had ED charges as a single predictor, and the third had the Elixhauser comorbidity variables reduced to its most parsimonious model. A subsequent logistic regression model was developed that included both 
APACHE-II score and ED charges, hoping to understand whether ED charges provided additional predictive power beyond that available with APACHE-II score alone.

Each continuous predictor was initially modeled as a categorical variable by dividing it into quintiles to test the assumption that the predictors were linearly associated with mortality. Once this assumption was confirmed, the final models used the original continuous variable as the predictor, if appropriate. These models were compared using the area under the curve (AUC) of the receiver operator characteristics analysis to compare the relative explanatory power of each index in predicting hospital survival.

For the secondary analysis, univariate linear regression models were constructed to measure the association between APACHE-II score and ED charges (separately) with 28-day hospital-free days and with 28-day ICU-free days.

We completed all analysis using Stata v. 13.1 (StataCorp, College Station, TX), and all results are reported using the Strengthening the Reporting of Observational Studies in Epidemiology (STROBE) guidelines [14].

\section{Availability of data and materials}

Because the data used for this analysis included identifiable protected health information, the original data set is not available for release.

\section{Results}

During the 2-year study period, 294 patients were included in the analysis, and 83 (28\%) died. Table 1 shows the demographics, ED interventions, comorbidities and baseline vitals. The median APACHE-II score was 17 (rang 2 to 47), and the median ED charge was \$3640 (IQR \$2861 - \$5234).

\section{Mortality}

ED charges were significantly associated with mortality, with higher ED charges being associated with lower mortality (adjusted OR 0.829 per $\$ 1000$ total ED charges, 95\%CI 0.702-0.980).

\section{Severity of illness}

Illness severity and ED charges were also associated ( $p=$ 0.016), with every point increase in APACHE-II score associated with a \$34 (95\% CI \$6-62) increase in ED charges (Fig. 1). Of the total ED charges, both hospital charges (\$20 per point increase in APACHE-II score, 95\%CI \$3-37) and professional fees (\$14 per point increase in APACHE-II score, 95\%CI \$2-26) were correlated with APACHE-II score.

\section{Prediction of mortality}

Elixhauser comorbidities, APACHE-II score, and ED charges all independently predict 28 -day mortality $(p<0.001, p=$ $0.010, \quad p=0.028)$. Elixhauser comorbidities predicted mortality well (AUC $=0.729$ ), while APACHE-II score and ED charges were significant but poor in their predictive value ( $\mathrm{AUC}=0.575$ and $\mathrm{AUC}=0.596$, respectively) (Fig. 2). Both APACHE-II and ED charges were linearly related to mortality.

\section{Prediction of length-of-stay}

ED charges were independently associated with 28-day hospital-free days ( 0.74 days increase in 28-day hospital-free days per $\$ 1000$ additional ED charges, 95\%CI 0.06-1.41) and 28-day ICU-free days (0.81 days increase in 28-day ICU-free days per $\$ 1000$ additional ED charges, 95\%CI 0.05-1.56). APACHE score was the stronger predictor of both hospital and ICU-free days (BIC 2188 vs. 2193 for hospital-free days, BIC 2254 vs. 2259 for ICU-free days).

\section{Discussion}

The ability to adjust for severity of illness is important in comparing clinical outcomes such as mortality and length-of-stay in observational health services analyses, but this adjustment has been elusive for administrative analyses of sepsis studies. The APACHE-II score is a robust and well-validated tool that can be used to predict mortality, and it is commonly used when comparing health care outcomes for critically ill patients [10]. Physiologic and laboratory data are often unavailable in health services analyses that are conducted using administrative claims data, which limits severity adjustment in these studies [15]. This study identifies ED charges as a potential surrogate measure, suggesting that it can be used as a covariate in outcomes-based sepsis studies using administrative claims, even when more robust physiologic severity of illness measures are not available.

ED charges are usually viewed as an outcome related to care provided, but they are also a reflection of the amount and type of care needed during an ED stay. In the United States, ED charges are generated from professional fees and facility fees, and charges performed during the study period were performed on a fee-for-service basis. These fees are based on the time that health care providers spend caring for a patient, separately billable procedures that are performed (e.g., central venous line placement, endotracheal intubation), and the medical complexity documented. All severity of illness indices are surrogate markers for the probability of death assessed at admission [9]. These markers are designed to allow for risk adjustment to measure the probability of death attributable to only conditions recognized at the time of hospital admission, and ED charges were hypothesized to function similarly. Patients who are more seriously ill (hypotensive, requiring intubation, or needing more time and resource-intensive care) also receive higher hospital bills, and that relationship was validated in our model. 
Table 1 Demographics and ED care for patients in study cohort. Baseline characteristics of the study population

\begin{tabular}{|c|c|c|c|c|}
\hline Factor & All Patients $(n=294)$ & Survivors $(n=211)$ & Non-survivors $(n=83)$ & Difference $(95 \% \mathrm{Cl})$ \\
\hline Male, n (\%) & $163(55)$ & $121(57)$ & $42(51)$ & $6.7(-6.0-19.4)$ \\
\hline Age, mean (SD) & $58.0(16.0)$ & $57.2(16.3)$ & $60.0(15.1)$ & $-2.6(-1.4-6.7)$ \\
\hline ICU Admission, n (\%) & $220(75)$ & $161(76)$ & $59(71)$ & $5.2(-5.9-16.3)$ \\
\hline Fluids Administered over 24 h, liters (mean, SD) & $4161(2881)$ & $4454(2940)$ & $3420(2596)$ & $1034(307-1761)$ \\
\hline Lactate, mmol/L (mean, SD) & $3.2(2.8)$ & $3.0(2.3)$ & $3.9(3.7)$ & $-0.9(-1.6-0.2)$ \\
\hline \multicolumn{5}{|l|}{ Triage Vital Signs } \\
\hline Triage systolic blood pressure, mmHg (mean, SD) & $108(65)$ & $109(74)$ & $106(31)$ & $3(-13-19)$ \\
\hline Triage heart rate, bpm (mean, SD) & $107(26)$ & $109(24)$ & $103(31)$ & $6(-1-12)$ \\
\hline White blood cell count, cells/mL (mean, SD) & $16.2(10.2)$ & $15.9(10.1)$ & $17.0(10.3)$ & $-1.1(-3.7-1.5)$ \\
\hline ED Charges, Total, dollars (median, IQR) & $\$ 3640(2861-5234)$ & $\$ 3840(2947-5661)$ & $\$ 3380(2793-4356)$ & $334(71-653)$ \\
\hline Hospital Facility Charges (median, IQR) & $\$ 2776(2232-3706)$ & $\$ 2863(2288-3772)$ & $\$ 2574(2049-3264)$ & $264(63-482)$ \\
\hline Professional Fees (median, IQR) & $\$ 760(660-1496)$ & $\$ 1050(681-1950)$ & $\$ 681(660-1050)$ & $19(0-21)$ \\
\hline \multicolumn{5}{|l|}{ Comorbidities } \\
\hline Peripheral vascular disease, n (\%) & $17(6)$ & $10(5)$ & $7(8)$ & $-3.7(-9.7-2.3)$ \\
\hline Paralysis, n (\%) & $17(6)$ & $14(7)$ & $3(4)$ & $3.0(-2.9-9.0)$ \\
\hline Neurologic disorders, n (\%) & $35(12)$ & $27(13)$ & $8(10)$ & $3.2(-5.1-11.4)$ \\
\hline COPD, n (\%) & $47(16)$ & $32(15)$ & $15(18)$ & $-2.9(-12.3-6.4)$ \\
\hline DM with complications, n (\%) & $23(8)$ & $20(9)$ & $3(4)$ & $5.9(-1.0-12.7)$ \\
\hline Hypothyroidism, n (\%) & $23(8)$ & $17(8)$ & $6(7)$ & $0.8(-6.0-7.7)$ \\
\hline Renal failure, n (\%) & $38(13)$ & $29(14)$ & $9(11)$ & $2.9(-5.7-11.5)$ \\
\hline Liver disease, n (\%) & $42(14)$ & $23(11)$ & $19(23)$ & $-12.0(-20.8--3.1)$ \\
\hline Metastatic cancer, n (\%) & $18(6)$ & $11(5)$ & $7(8)$ & $-3.2(-9.3-2.9)$ \\
\hline Solid tumor without metastasis, n (\%) & $16(5)$ & $8(4)$ & $8(10)$ & $-5.8(-11.6--0.1)$ \\
\hline Coagulopathy, n (\%) & $53(18)$ & $30(14)$ & $23(28)$ & $-13.4(-23.2--3.7)$ \\
\hline Fluid and electrolyte disorders, n (\%) & $231(79)$ & $153(73)$ & $78(94)$ & $-21.4(-31.7--11.3)$ \\
\hline Deficiency anemia, n (\%) & $34(12)$ & $30(14)$ & $4(5)$ & $9.4(1.2-17.5)$ \\
\hline Hypertension, n (\%) & $96(32)$ & $7(3)$ & $5(6)$ & $17.0(5.1-28.8)$ \\
\hline APACHE-II score (mean, SD) & $16.9(7.0)$ & $16.2(6.5)$ & $18.6(7.8)$ & $-2.4(-4.1--0.6)$ \\
\hline 28-day ICU-free days (median, IQR) & $22(0-26)$ & $25(22-26)$ & $0(0-0)$ & $25(24-25)$ \\
\hline 28-day hospital free days (median, IQR) & $12(0-21)$ & $18(10-22)$ & $0(0-0)$ & $18(17-20)$ \\
\hline
\end{tabular}

In addition to finding that ED charges are related to mortality, however, the decreased mortality was found with higher charges. This finding might indicate that care delivered in the ED (measured by charges) may also be influencing outcomes. Early aggressive ED sepsis care has been shown to decrease mortality [16], and the higher ED charges may be capturing the impact of aggressive appropriate resuscitation. This finding suggests that ED charges may be included in studies of non-ED interventions on sepsis outcomes, because controlling for ED charges may capture both the effect of severity of illness and treatment effects of ED care.

This study introduces ED charges as a predictor of clinical outcomes when physiologic severity of illness scores are not available retrospectively for research purposes. There are many areas of acute care research that may benefit from adjusting for ED charges as a marker of disease severity. Studies of diseases where acute disease severity strongly influences outcomes, such as sepsis, acute respiratory failure, stroke, and trauma may benefit from such an adjustment. Although these data only inform the use of ED charges in sepsis studies, other future studies should consider ED charges for its predictive value. In this way, ED charges can be treated as a nuisance variable - a variable that may be associated with a potentially confounding variable, but where the magnitude of effect is irrelevant in itself. There may also be other non-critical care diseases where this adjustment may also be useful, such as asthma or congestive heart failure. 


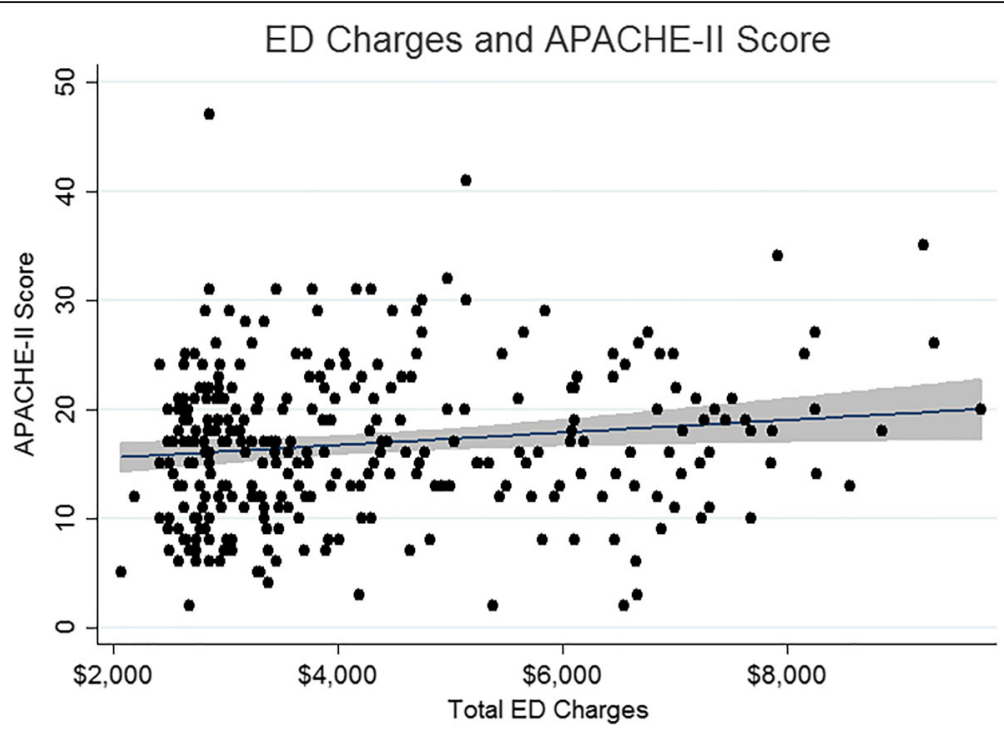

Fig. 1 Scatter plot comparing ED charges with APACHE-II scores

\section{Limitations}

This study has several limitations. First, we used retrospectively collected data from a single site. Although this may limit the external validity of our finding, it reflects accurately the way that this tool may be used in actual observational analyses. For our data collection we looked at ED charges, not actual cost to the patient. Costs are challenging to estimate, so they often are estimated based on the cost-tocharge ratio, and use of this ratio would be expected to yield similar precision. As with all claims-based studies, there is a bias towards identifying patients with more severe illness [17]. The high mortality in this cohort, however, supports that severity was likely reflected accurately. Finally, as a single-center study, we have no data on the concordance between hospitals, which is an important consideration for this method to be used on a large scale. Additionally, the application of ED cost to predict mortality may not be applicable outside of the United States where charges are more variable. Fortunately, studies that propose to use this method can validate the association between ED charges and clinical outcomes within the study, so using ED charges as a covariate in a multivariable regression model will allow one to assess its contribution to model fit in each application independently.

This study also included only patients admitted to an intensive care unit. These inclusion criteria were intended to

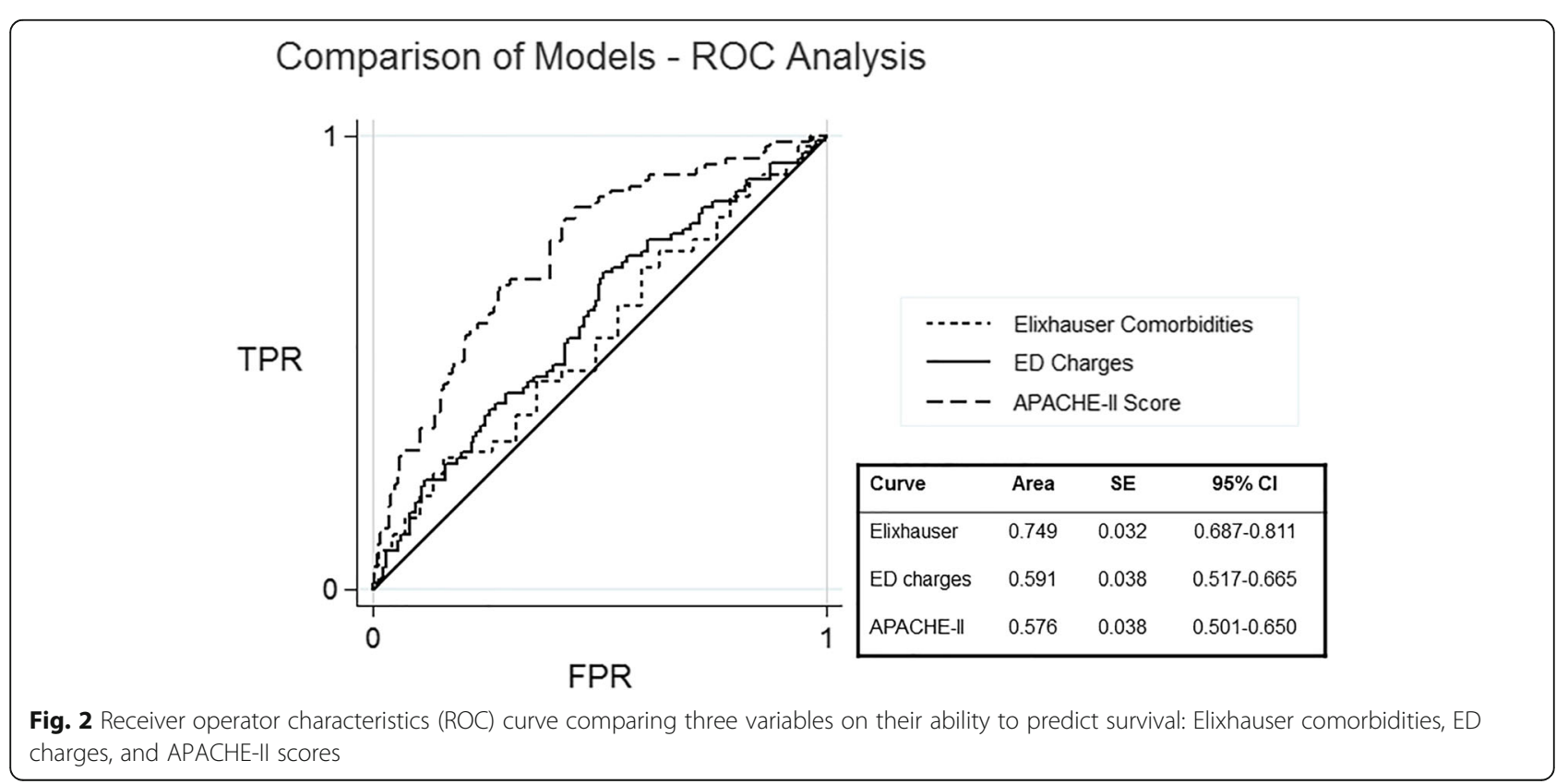


limit the study population to those with greater severity of illness, but it may limit the generalizability of the findings.

\section{Conclusions}

ED charges are a useful surrogate measure for severity of illness in sepsis studies based on administrative data. ED charges are inversely associated with mortality in severe sepsis and septic shock, and they predict hospital and ICU length-of-stay. ED charges may reflect both severity of illness and the contribution of ED care on clinical outcomes. Future work should focus on the applicability of comparing this covariate across hospital systems to adjust severity of illness in large administrative data sets, and to use it in other disease categories.

\section{Abbreviations \\ APACHE-II: Acute Physiology and Chronic Health Evaluation, 2nd edition; AUC: Area Under the Curve; BIC: Bayesian Information Criteria; ED: Emergency Department; ICD-9: International Classification of Diseases, 9th Edition; ICU: Intensive Care Unit; SAPS: Simplified Acute Physiology Score; SOFA: Sequential Organ Failure Assessment; STROBE: Strengthening the Reporting of Observational Studies in Epidemiology}

\section{Funding}

This project is supported by the Emergency Medicine Foundation, the University of lowa Department of Emergency Medicine, and the University of lowa Institute for Clinical and Translational Sciences, which is supported by the National Institutes of Health (NIH) Clinical and Translational Science Award (CTSA) program, grant U54TR001356. The organizations funding this research did not have any access to data or contribute to the study design or interpretation. The funding organization played no role in the acquisition, analysis, or reporting of this research report. Dr. Mohr is also supported by a grant from the Federal Office of Rural Health Policy. Dr. Fuller was funded by the Washington University KL2 Career Development Award and (KL2 TR000450) from the National Center for Advancing Translational Sciences (NCATS) and the Foundation for Barnes-Jewish Hospital Clinical and Translational Sciences Research Program (Grant \# 8041-88).

\section{Availability of data and materials}

The datasets analyzed during the current study are available in de-identified form from the corresponding author on reasonable request.

\section{Authors' contributions}

NMM, RDP, and AA conceived the study, interpreted the analysis, and drafted the manuscript. KKH conducted the analysis, interpreted the analysis, and drafted critical portions of the manuscript. DS, BMF, and JCT contributed to interpretation of the study findings and critically revised the manuscript. DM, CM, and LK collected the research data, contributed to interpretation of the analysis, and critically revised the manuscript. All authors read and approved the final manuscript.

\section{Ethics approval and consent to participate}

This study was approved by the University of lowa Institutional Review Board (IRB \# 201211702) under waiver of informed consent.

\section{Consent for publication}

Not applicable (no individually identifiable patient data are contained in this manuscript)

\section{Competing interests}

The authors declare that they have no competing interests.

\section{Publisher's Note}

Springer Nature remains neutral with regard to jurisdictional claims in published maps and institutional affiliations.

\section{Author details}

'Department of Emergency Medicine, University of lowa College of Medicine, 200 Hawkins Drive, 1008 RCP, lowa City, IA 52246, USA. ²Division of Critical Care, Department of Anesthesia, University of lowa Carver College of Medicine, 200 Hawkins Dr. 6546 JCP, lowa City, IA 52246, USA. ${ }^{3}$ Department of Health Management and Policy, University of lowa College of Public Health, N244 CPHB, lowa City, IA 52246, USA. "TeamHealth, St. Rose Dominican Hospital-Siena Campus, Southern Hills Hospital and Medical Center, 2380 W. Horizon Ridge Parkway, Ste 110, Henderson, NV 89052, USA. ${ }^{5}$ Division of Critical Care, Department of Anesthesiology, Division of Emergency Medicine, Washington University School of Medicine, One Brookings Drive, St. Louis, MO 63130, USA. ${ }^{6}$ Department of Epidemiology, University of lowa College of Public Health, S441A CPHB, lowa City, IA 52246, USA.

Received: 2 May 2018 Accepted: 7 December 2018

Published online: 29 December 2018

\section{References}

1. Kilgannon J, Jones $\mathrm{AE}$, Shapiro NI, et al. Association between arterial hyperoxia following resuscitation from cardiac arrest and in-hospital mortality. JAMA. 2010:303(21):2165-71.

2. Tirschwell DL, Longstreth WT Jr. Validating administrative data in stroke research. Stroke. 2002;33(10):2465-70.

3. Angus DC, van der Poll T. Severe Sepsis and septic shock. N Engl J Med 2013:369(9):840-51.

4. Rubenfeld GD, Caldwell E, Peabody E, Weaver J, Martin DP, Neff M, et al. Incidence and outcomes of acute lung injury. N Engl J Med. 2005;353(16):1685-93.

5. Rothberg MB, Pekow PS, Priya A, Zilberberg MD, Belforti R, Skiest D, et al. Using highly detailed administrative data to predict pneumonia mortality. PLoS One. 2014;9(1).

6. Strehlow MC, Emond SD, Shapiro NI, Pelletier AJ, Camargo CA, Jr. National study of emergency department visits for sepsis, 1992 to 2001. Ann Emerg Med 2006;48(3):326-331, 31.e1-3.

7. Berger T, Green J, Horeczko T, Hagar Y, Garg N, Suarez A, et al. Shock index and early recognition of Sepsis in the emergency department: pilot study. West J Emerg Med. 2013;14(2):168-74.

8. Thomas JW. Risk adjustment for measuring health care outcomes, 3rd edition. Int J Qual Health Care. 2004:16(2):181-2.

9. Knaus WA, Zimmerman JE, Wagner DP, Draper EA, Lawrence DE. APACHEacute physiology and chronic health evaluation: a physiologically based classification system. Crit Care Med. 1981;9(8):591-7.

10. Knaus WA, Draper EA, Wagner DP, Zimmerman JE. APACHE II: a severity of disease classification system. Crit Care Med. 1985;13(10):818-29.

11. Jones $A E$, Trzeciak S, Kline JA. The sequential organ failure assessment score for predicting outcome in patients with severe sepsis and evidence of hypoperfusion at the time of emergency department presentation. Crit Care Med. 2009:37(5):1649-54

12. Moreno RP, Metnitz PG, Almeida E, Jordan B, Bauer P, Campos RA, et al. SAPS 3--from evaluation of the patient to evaluation of the intensive care unit. Part 2: development of a prognostic model for hospital mortality at ICU admission. Intensive Care Med. 2005;31(10):1345-55.

13. Elixhauser A, Steiner C, Harris DR, Coffey RM. Comorbidity measures for use with administrative data. Med Care. 1998;36(1):8-27.

14. von Elm E, Altman DG, Egger M, Pocock SJ, Gotzsche PC, Vandenbroucke $J P$. The strengthening the reporting of observational studies in epidemiology (STROBE) statement: guidelines for reporting observational studies. Lancet (London, England). 2007;370(9596):1453-7.

15. Polderman KH, Girbes AR, Thijs LG, Strack van Schijndel RJ. Accuracy and reliability of APACHE II scoring in two intensive care units problems and pitfalls in the use of APACHE II and suggestions for improvement. Anaesthesia. 2001;56(1):47-50.

16. Rivers E, Nguyen B, Havstad S, Ressler J, Muzzin A, Knoblich B, et al. Early goal-directed therapy in the treatment of severe Sepsis and septic shock. N Engl J Med. 2001;345(19):1368-77.

17. Whittaker SA, Mikkelsen ME, Gaieski DF, Koshy S, Kean C, Fuchs BD. Severe sepsis cohorts derived from claims-based strategies appear to be biased toward a more severely ill patient population. Crit Care Med. 2013:41(4):945-53. 